\title{
Spectrum of microbial growth and antimicrobial usage in an intensive-care unit of a tertiary-care hospital in Trinidad, West Indies
}

\author{
S Bidaisee, MBBS, DM; S Hariharan, MBBS, MD, PhD, FCCM; D Chen, MBBS, FRCA, MBA \\ Anaesthesia and Intensive Care Unit, Faculty of Medical Sciences, University of the West Indies, St. Augustine, Trinidad and Tobago
}

Corresponding author: S Hariharan (uwi.hariharan@gmail.com)

\begin{abstract}
Background. Intensive-care units (ICUs) are a source of multidrug-resistant organisms, owing to the indiscriminate usage of broad-spectrum antimicrobial drugs. In such settings, one must be aware of the spectrum of microbes and pattern of antibiotic usage.

Objectives. To evaluate the spectrum, susceptibility and resistance patterns of microbes found in ICU patients in a tertiary-care teaching hospital in Trinidad, and to quantify antimicrobial usage.

Methods. All adult patients ( $\geq 15$ years of age) admitted to the ICU for $\geq 48 \mathrm{~h}$ who developed nosocomial infections conforming to the Centers for Disease Control and Prevention criteria were included. Demographic data and clinical data, including specimens sent, isolates grown, antimicrobial sensitivity and resistance patterns, the usage of antimicrobials and patient outcomes, were recorded. Variables such as age, admission white blood cell count, duration of first antibiotic used, length of ICU stay, length of hospital stay, organ support and total comorbidities were analysed. Antimicrobial usage was quantified as the defined daily dosage per 1000 patient-days.

Results. A total of 153 patients with 287 microbiological specimens were studied. The mean patient age was 48.4 years, and the mean ICU length of stay was 7.9 days. The most common admitting diagnoses were sepsis and multiple trauma. Staphylococcus aureus was the most common isolate from blood and central venous lines, and Pseudomonas aeruginosa from tracheal aspirates and wound swabs. Non-survivors had significantly higher age, leucocyte count and organ support requirements, and shorter lengths of stay. Cefuroxime was the most-used antimicrobial in the unit. Conclusion. The usage pattern of antimicrobials did not correlate with susceptibility in most instances. There is a need to improve antimicrobial usage by implementing antimicrobial-stewardship programmes to establish an antimicrobial protocol and guidelines for usage in the ICU.
\end{abstract}

S Afr J Crit Care 2017;33(1):39-44. DOI:10.7196/SAJCC.2017.v33i2.284

Hospital-acquired infections (HAIs) are a major cause of morbidity and mortality in patients admitted to hospitals, and can lead to the spread of multidrug-resistant pathogens. ${ }^{[1]}$ HAIs, by definition, may be either local and/or systemic, and result from the presence of an infectious agent or its toxin after 48 hours or more following a hospital admission, where the incubation period for the infection does not occur prior to hospital admission. ${ }^{[2]}$ Over the years, patterns of microbial growth have seen many shifts, with emerging strains of potential drugresistant species, including methicillin-resistant Staphylococcus aureus (MRSA), Pseudomonas spp., Acinetobacter spp., Stenotrophomonas maltophilia and extended-spectrum beta-lactamase (ESBL)-producing Gram-negative organisms. ${ }^{[3]}$

Inappropriate antimicrobial use and poor prescription practices have contributed to the development of multidrug-resistant organisms, rendering treatment of HAIs very difficult. ${ }^{[4]}$ In addition, failure to comply with infection-control measures has a negative impact on hospital infection rates. Overcrowding and understaffing of wards also contribute to HAIs in the developing world. In low- and middleincome countries, the major factors contributing to serious nosocomial infections include lack of infrastructure, inconsistent surveillance, deficiency in trained personnel and infection-control programmes, and poverty-related factors. ${ }^{[5]}$

Intensive-care patients are often the most susceptible to HAIs, due to the acute disease process, the presence of comorbidities, invasive devices, exposure to various procedures and general immunocompromised status. ${ }^{[6]}$ Therefore microbial surveillance and knowledge of resistance patterns to antimicrobials is critically important in any ICU setting. A recent review showed that the implementation of continuous surveillance measures had a positive impact in controlling nosocomial infections. ${ }^{[7]}$ Although surveillance programmes may be relatively expensive to implement, they are indeed cost-effective when compared to the expenditure of treating sepsis and the morbidity associated with it. Surveillance studies also play a vital part in the optimal management of infections in the intensive-care setting. Many developed countries have well-established surveillance teams and programmes that have accounted for lower infection rates when compared with less-developed countries. ${ }^{[8]}$ In the USA, the Centers for Disease Control and Prevention (CDC) launched a subset division called the National Nosocomial Infections Surveillance System in 2004, and publish yearly benchmarks for the surveillance measures ${ }^{[9]}$ Such surveillance, along with intervention with prevention strategies, can decrease infection rates, morbidity and mortality, increasing patient safety.

The International Nosocomial Infection Control Consortium is an international collaborative body founded 10 years ago to promote infection control in the hospitals of countries with limited resources. ${ }^{[10]}$ Thus far, it has been successful in increasing compliance rates for prevention strategies and has reduced the rates of HAI. ${ }^{[11]}$ Trinidad and Tobago, unfortunately, has not been part of any such international programme. 
Against this background, this study aimed to look at the microbial growth patterns, susceptibility profiles, resistance patterns and antimicrobial usage in the ICU at Eric Williams Medical Sciences Complex, a major tertiary-care teaching health facility in Trinidad.

\section{Design and methods Study setting}

Trinidad is one half of the twin island-nation of Trinidad and Tobago, with a population of approximately 1.3 million. The study hospital has 323 beds and the Intensive Care Unit (ICU) is a 10-bed mixed surgical and medical ICU. Admissions to the ICU are either directly from the emergency departments, the operating theatres, the High Dependency Unit or the general wards. The ICU team consists of an anaesthesia consultant, a registrar and two house officers. Although the aim is to have a nurse-to-patient ratio of 1:1, sometimes it happens to be 1:2. Four beds are in isolation rooms at the back of the ICU, and are designated for barrier nursing. An infection prevention and control officer visits the ICU on a daily basis.

\section{Description of the study}

Approval for this retrospective observational study was obtained from the Ethics Committee of the Faculty of Medical Sciences, University of the West Indies, with a waiver of individual informed patient consent. Approval was also given by the medical chief of staff and the head microbiologist at the hospital.

All adult patients ( $\geq 15$ years of age) admitted to the ICU between January 2008 and June 2010 for $\geq 48 \mathrm{~h}$ who developed nosocomial infections meeting the $\mathrm{CDC}$ criteria were included. Paediatric patients (i.e. $<15$ years) and patients admitted for $<24 \mathrm{~h}$ were excluded.

\section{Data collection}

The demographic data on patient age and gender were collected. The clinical data collected included the following:

- admission diagnosis

- comorbidities

- body temperature on admission

- leukocyte count on admission

- culture sites/specimens sent

- isolates grown from specimens

- sensitivity profiles and resistance patterns for each isolate

- antimicrobial agent(s) used in each patient throughout the stay, including the dosage and duration of use

- length of stay in ICU and hospital

- types of organ support provided in the ICU - mechanical ventilation for the respiratory system, renal-replacement therapy for acute kidney injury, haemodynamic support (pharmacological/intra-aortic balloon pump, etc.) for the cardiovascular system.

- patient outcome - death or discharge from ICU.

In this hospital, during the study period, the ICU did not have a surveillance policy. Specimens were taken from patients based on clinical requirements as ordered by the intensivists. In addition, the microbiology laboratory did not have an international policy manual to follow. The testing was done based on guidelines proposed by the consultant microbiologist, which were based on those recommended by the American Society for Microbiology. The study samples were processed as follows:

As per hospital general procedure, blood-culture samples taken on wards are placed in either aerobic or anaerobic bottles. In the laboratory, these samples are placed in a Bactec 9240 (BD, USA) machine set at $35^{\circ} \mathrm{C}$, where they are rotated. Once $\mathrm{CO}_{2}$ is detected by the machines, the bottles are removed and set for Gram stain and plating. The samples are plated on different media (blood, chocolate agar, MacConkey agar, anaerobic agar). Each medium is set at specific temperatures and left for 5 days to observe for growth, but they are monitored on a 24-hour basis.

Catheter tips (e.g. central venous catheter (CVC) tips) are rubbed onto the plates and streaked. Any wet material present on the catheter is also Gram stained. Urine samples obtained are either catheter or midstream samples, and either they are placed in a calibrated loop, or the appropriate quantity of urine is placed on a cystine lactose electrolyte deficient (CLED) plate to observe for growth. Tracheal aspirates are obtained from suctioning of a patient's trachea with inline suction traps. In the laboratory, they are plated on blood, or MacConkey or chocolate agar. The isolates are then tested for antimicrobial susceptibility using disks which have been incubated overnight, and the minimum inhibitory concentration is estimated in accordance with the standards of the Clinical and Laboratory Standards Institute.

\section{Data analysis}

Data were entered into Excel spreadsheets and Statistical Package for Social Sciences (SPSS) version 12 (IBM, USA) was used for statistical analyses. Statistical significance was fixed at $p<0.05$. Descriptive analyses were used for demographic data, and independent $t$-tests were used to compare the variables such as age, admission white blood cell count (WBC), duration of first antibiotic used, length of ICU stay, length of hospital stay, organ support and total comorbidities between patients who died in the ICU and those who survived.

Antimicrobial usage was represented as defined daily dosage (DDD) per 1000 patient-days (using the following formula:

$$
\mathrm{DDD}=\frac{\mathrm{DDD} \text { for specific agent }(\mathrm{ATC} / \mathrm{DDD} \text { Code })}{\text { Total number of patient-days }} \times 1000
$$

\section{Results}

A total of 153 patients were included in the study, 79 (51.6\%) of whom were female. The most common admitting diagnoses were multiple trauma and neurological disorders, and the most common comorbidities were diabetes mellitus, hypertension and ischaemic heart disease. Fig. 1 depicts the distribution of the diagnoses on admission to ICU.

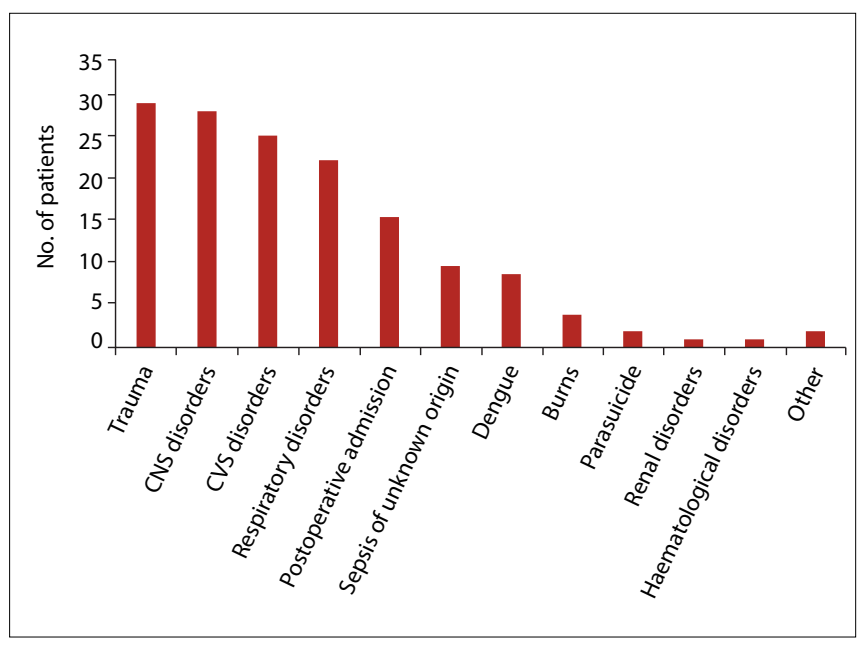

Fig. 1. Distribution of diagnoses on admission. (CNS = central nervous system; CVS $=$ cardiovascular system .) 
The age of patients ranged from 16 to 90 years. The mean (standard deviation (SD)) age of patients was 48.4 (18.7) years (range 16 - 90) and the mean (SD) WBC on admission was 12.9 (5.9) (range 4.2 - 40.9). The mean (SD) length of ICU stay was 7.9 (5.9) (range 2 - 33) days, while the mean (SD) overall length of hospital stay was 15.3 (9.7) (range 2 - 65) days.

The comparison between patients who survived their ICU stay and those who died is shown in Table 1. The length of ICU stay and duration of first antibiotic use did not show a statistically significant difference between the two groups. With respect to age, the survivors were younger than those who died $(p=0.01)$. The non-survivors had a higher WBC count on admission when compared with those who survived ( $p=0.02$ ). As expected, the patients who had a higher number of comorbidities and required more organ support had a higher mortality $(p<0.001)$.

Of all the patients, only two did not require organ support, and were admitted for close monitoring of vital signs. The majority (62.7\%) of patients received support for one organ in the form of mechanical ventilation and $33.3 \%$ received support for two organs. The most common (96.7\%) organ system supported was the respiratory system. Of these, 3 patients (1.9\%) required non-invasive positive-pressure ventilation, while the remainder required invasive ventilation. Inotropic support was required in 52 patients (33.9\%), while 3 patients (1.9\%) needed renal replacement therapy (RRT). While 107 patients $(69.9 \%)$ were discharged from the ICU, 46 (30.1\%) died.

In total, 88 patients had at least one isolate in one of the specimens sent to the microbiology laboratory, meaning that the prevalence rate of nosocomial infections in this ICU was $57.5 \%$. The total number of specimen samples sent for microbiological analysis during the study was 282. The various specimens included blood, tracheal aspirates, urine, CVC tips, wound swabs, urinary catheter tips and pleural fluid. Wound swabs had the highest prevalence of microbial growth (95\%), followed by tracheal aspirates $(78 \%)$. Blood was the most common sample site to be cultured, accounting for 105 specimens (36.6\%).

The isolates grown from the different specimens are shown in Table 2. In general, Pseudomonas aeruginosa and Enterobacter spp. were reported in all the various sites and specimens sampled for microbiological analyses, while Staphylococcus aureus was reported in all except urine.

P. aeruginosa was predominantly sensitive to gentamicin (63.1\%), as well as to ciprofloxacin (60.1\%). It showed $37 \%$ sensitivity to piperacillin/ tazobactam. Sensitivity to imipenem was $32 \%$ while meropenem was at $13 \%$. It was least sensitive to ceftazidime (8\%). Fig. 2 shows the individual sensitivity and resistance pattern of this organism. Fig. 3 shows the sensitivity profile for Klebsiella, and of particular note is that $5 \%$ of these isolates were resistant to meropenem.

Fig. 4 shows the sensitivity-resistance pattern of $S$ aureus. A total of $42 \%$ of these isolates were sensitive to oxacillin, amoxicillin/lavulanic acid and gentamicin.

S. epidermidis showed highest sensitivity to gentamicin, followed by levofloxacin. Sensitivity to cefuroxime was $19 \%$, while that for cefotaxime was $3 \%$. Resistance to gentamicin was at $19 \%$, while that for piperacillin/tazobactam and imipenem was $6 \%$ each.

Enterobacter spp. showed $75 \%$ sensitivity to gentamicin. Sensitivity to ciprofloxacin was at $60 \%$ and piperacillin/tazobactam at $50 \%$. Sensitivity to cefotaxime and ceftazidime were at $5 \%$ each. Resistance was highest for ciprofloxacin and lowest for gentamicin at $5 \%$.

S. haemolyticus showed no resistance to the cephalosporins, gentamicin or vancomycin.

Methicillin-resistant S. aureus was sensitive to linezolid (71\%), tigecycline $(57 \%)$ and rifampin (43\%). There was no vancomycinresistant specimen in this study.

Table 1. Comparison of patient variables between survivors and non-survivors

\begin{tabular}{lllll}
\hline Variable & Overall, mean (SD) & Survivors, mean (SD) & Non-survivors, mean (SD) & $\boldsymbol{p}_{\text {-value }}^{*}$ \\
\hline Age (years) & $48.4(18.7)$ & $45.7(18.3)$ & $54.7(18.4)$ & 0.01 \\
Admission leukocyte count $\left(\times 10^{3} / \mu \mathrm{L}\right)$ & $13.0(5.9)$ & $12.3(4.9)$ & $14.6(7.4)$ & 0.02 \\
Hospital length of stay (days) & $15.3(9.7)$ & $16.9(9.8)$ & $11.8(8.7)$ & 0.002 \\
Organ support $(n)$ & $1.3(0.5)$ & $1.2(0.5)$ & $1.7(0.6)$ & $<0.001$ \\
Total comorbidities $(n)$ & $1.57(0.8)$ & $1.48(0.8)$ & $1.78(0.8)$ & 0.04 \\
Duration of first antibiotic (days) & $6.9(3.9)$ & $6.9(3.6)$ & $6.8(4.6)$ & 0.855 \\
*Statistical significance by independent $t$-test. & & & &
\end{tabular}

Table 2. Isolates from various specimens

\begin{tabular}{|c|c|c|c|c|c|}
\hline Organisms identified & $\begin{array}{l}\text { Blood } \\
(N=105)\end{array}$ & $\begin{array}{l}\text { Tracheal aspirate } \\
(N=94)\end{array}$ & $\begin{array}{l}\text { Urine } \\
(N=43)\end{array}$ & $\begin{array}{l}\text { CVC } \\
(N=20)\end{array}$ & $\begin{array}{l}\text { Wound swabs } \\
(N=20)\end{array}$ \\
\hline Total isolates & 51 & 73 & 13 & 14 & 19 \\
\hline Sterile (no bacterial growth) & $54(51.6)$ & $21(22.3)$ & $30(69.8)$ & $6(30.0)$ & $1(5.0)$ \\
\hline Pseudomonas aeruginosa & $4(7.8)$ & $24(32.8)$ & $1(7.7)$ & $2(14.3)$ & $8(42.1)$ \\
\hline Staphylococcus aureus & $7(13.7)$ & $4(5.5)$ & $2(15.4)$ & - & $2(10.5)$ \\
\hline S. haemolyticus & $8(15.6)$ & $1(1.3)$ & - & $1(7.1)$ & - \\
\hline S. epidermidis & $23(45.1)$ & $1(1.3)$ & $1(7.7)$ & $8(57.1)^{*}$ & - \\
\hline Enterobacter spp. & $4(7.8)$ & $11(15.1)$ & $2(15.4)$ & $1(7.1)$ & $1(5.2)$ \\
\hline Klebsiella & $5(9.8)$ & $13(16.7)$ & $2(15.4)$ & - & $2(10.5)$ \\
\hline MRSA & - & $5(6.8)$ & - & $1(7.1)$ & $3(15.8)$ \\
\hline Candida spp. & - & $1(1.3)$ & $4(30.8)$ & - & - \\
\hline Other & - & $13(17.8)^{\dagger}$ & - & $1(7.1)^{\ddagger}$ & $3(15.8)^{\S}$ \\
\hline
\end{tabular}




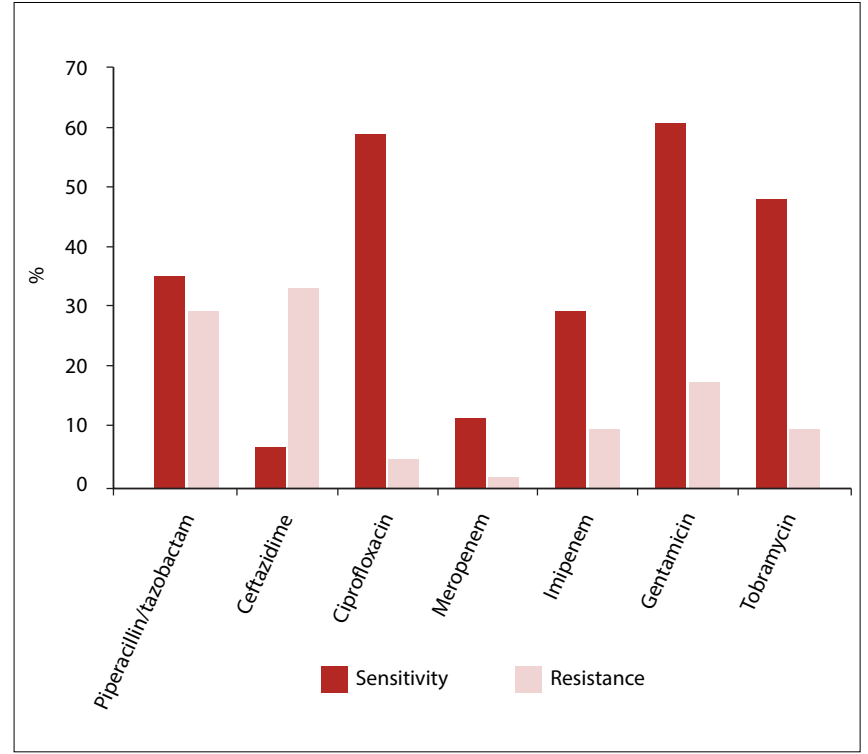

Fig. 2. Sensitivity-resistance patterns for Pseudomonas aeruginosa.

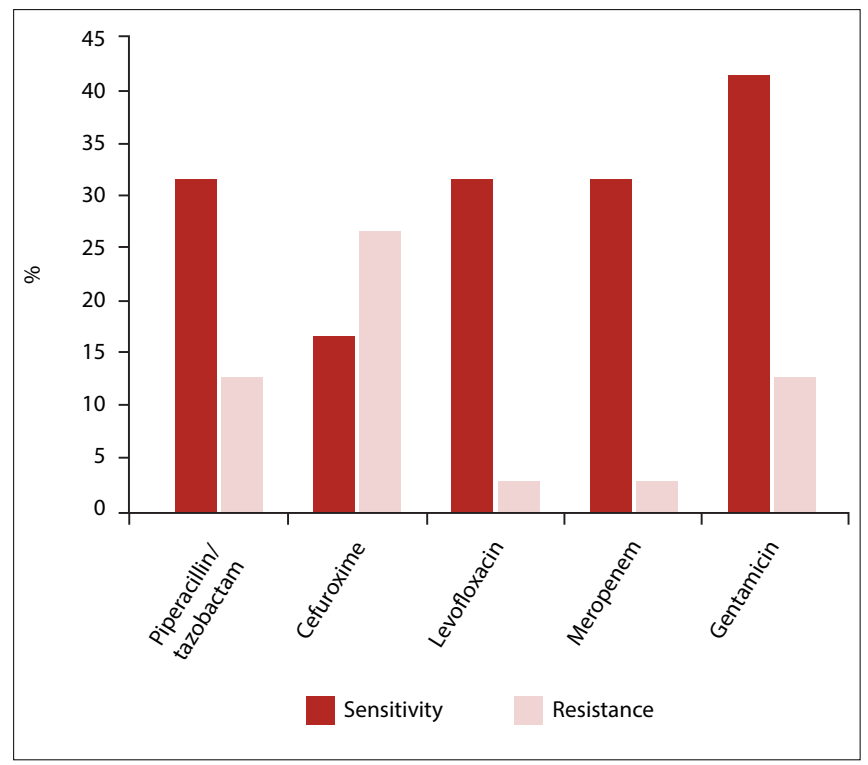

Fig. 3. Sensitivity-resistance patterns for Klebsiella.

Acinetobacter showed high sensitivity to piperacillin/tazobactam, ceftazidime, imipenem and tobramycin, gentamicin and levofloxacin; however, only $20 \%$ each of the isolates showed sensitivity to meropenem and ceftazidime.

S. maltophilia was isolated in two blood specimens. On both occasions it was sensitive to gentamicin (100\%), but only once to amikacin and levofloxacin (50\% each). There was $100 \%$ resistance to tobramycin.

The antimicrobial usage during the study period is depicted in Table 3 as the DDD per 1000 patient-days, along with the respective anatomical therapeutic chemical (ATC) classification and DDD code. The most common antibiotic prescribed in this study group was cefuroxime. Piperacillin/tazobactam, meropenem and ceftriaxone were the second, third and fourth most commonly used antimicrobials, respectively.

Antibiotic usage ranged from one agent to a maximum of four agents per patient throughout ICU stay, and the ranges of duration of antibiotic use are shown in Table 4. Monotherapy was used in 51 patients (33.1\%), while 57 patients (37.3\%) were given two antibiotics. Three antibiotics

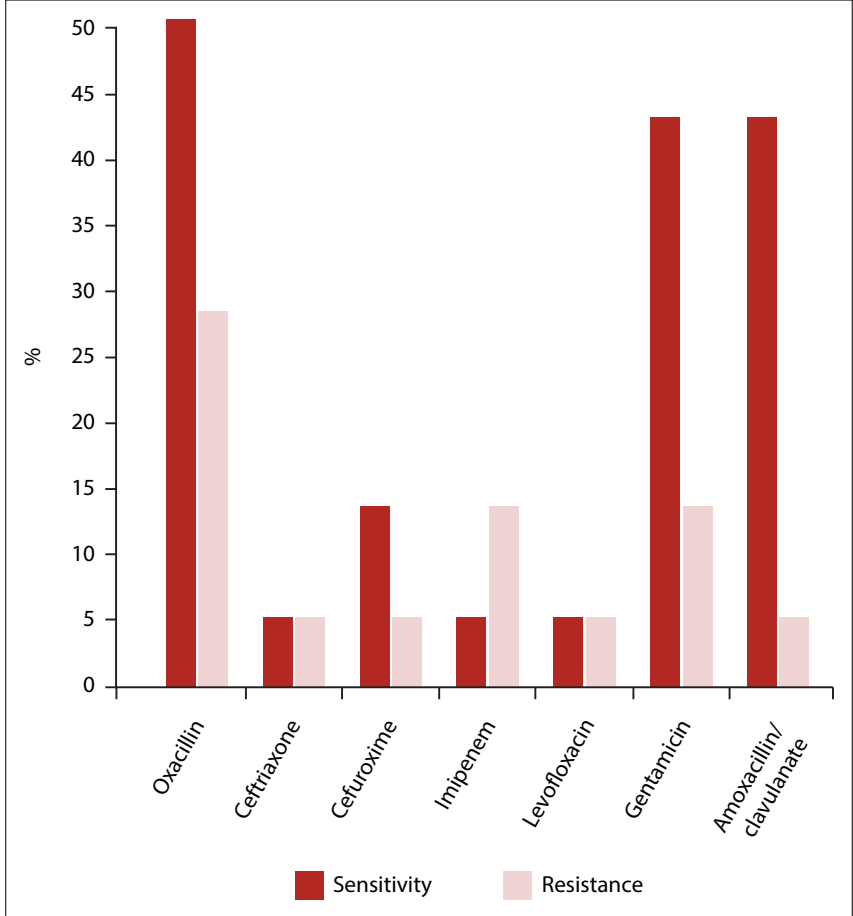

Fig. 4. Sensitivity-resistance patterns for Staphylococcus aureus.

Table 3. Antimicrobial usage as DDD per 1000 patient-days

\begin{tabular}{lll}
\hline Antimicrobial & ATC/DDD code & DDD \\
\hline Cefuroxime & J01DCO2 & 483.66 \\
Piperacillin/tazobactam & J01CR05 & 433.67 \\
Meropenem & J01DHO2 & 418.06 \\
Ceftriaxone & J01DD04 & 305.55 \\
Metronidazole & J01XD01 & 261.44 \\
Levofloxacin & J01MA12 & 170.75 \\
Azithromycin & J01FA10 & 101.67 \\
Gentamicin & J01GB03 & 98.66 \\
Cefotaxime & J01DDO1 & 46.29 \\
Vancomycin & J01XA01 & 40.52 \\
Augmentin & J01CR02 & 24.65 \\
Erythromycin & J01FA01 & 22.70 \\
Imipenem & J01DH51 & 19.61
\end{tabular}

DDD $=$ defined daily dosage; $\mathrm{ATC}=$ anatomical therapeutic chemical .

Table 4. Comparison of antibiotic usage

\begin{tabular}{lll}
\hline Category & $\begin{array}{l}\text { Duration, } \\
\text { range (days) }\end{array}$ & $\begin{array}{l}\text { Mean (SD), } \\
\text { (days) }\end{array}$ \\
\hline 1st antibiotic $(n=153)$ & $1-21$ & $6.92(3.9)$ \\
2nd antibiotic $(n=96)$ & $1-39$ & $7.5(5.1)$ \\
3rd antibiotic $(n=32)$ & $1-14$ & $5.6(3.0)$ \\
4th antibiotic $(n=8)$ & $2-10$ & $5.13(2.4)$ \\
SD $=$ standard deviation. & &
\end{tabular}

were used in 37 patients (24.7\%), while 8 patients (5.2\%) received the maximum of 4 antibiotics.

All patients admitted to the ICU received antibiotics, irrespective of their infective status. Since the antimicrobial choice was made by the clinicians (including the parent units as well as the intensivists) devoid of a standard protocol, it was difficult to quantitate the inappropriate use of antimicrobials. There were no correlations between the culture and 
sensitivity reports and the usage of antimicrobials in individual patients, and there was no evidence of de-escalation of antimicrobials in any patient during the study period.

\section{Discussion}

Surveillance of microbes and their resistance patterns is invaluable in infection control and prevention in hospital medicine. Data regarding microbial spectrum and antimicrobial usage in different settings also help to compare these factors in developed and developing countries. The present study was able to achieve these objectives.

The demographics of the patients in the present study were similar to those in a study from the neighbouring island Barbados. ${ }^{[12]}$ There was a significant relationship between increased WBC and mortality in this study. WBC has been shown to be a clinical marker of inflammation and infection, and is considered an independent predictor of all-cause mortality in a previous report. ${ }^{[13]}$

Increased length of ICU stay is generally associated with increased infection rates, contributing to higher mortality risk and increased cost of illness. ${ }^{[1,15]}$ This study did not, however, show a significant difference in this regard. Overall, the length of ICU stay of patients in the present study was comparable to that of previous reports originating from Barbados and China. ${ }^{[12,16]}$

Blood was the most frequently sent specimen for bacteriological culture in the present study. This differs from the Barbados study, where tracheal aspirates were the most common specimens. ${ }^{[12]}$ Unlike in the Barbados research, in the present study not all patients were routinely sampled for microbiological cultures on admission, and many patients did not have such investigations during their whole ICU stay, unless signs of respiratory infection were evident, such as a change in the nature of tracheal secretions or radiological evidence suggestive of chest infection.

The majority of the tracheal aspirates yielded Gram-negative bacilli. $P$. aeruginosa was one of the most common organisms, as was found by a study from the USA that reported Pseudomonas and Klebsiella as the most common organisms. ${ }^{[17]} \mathrm{A}$ report from Serbia also found Gram-negative organisms such as Pseudomonas and Acinetobacter to be the most common organisms in hospital-acquired pneumonias. ${ }^{[18]}$ Studies from ICUs in Egypt and India found Klebsiella to be the most common organisms associated with ventilator-associated pneumonia (VAP) ${ }^{[19,20]}$ The ICU where the present study was conducted does not formally adhere to any 'ventilator care bundle', and VAP rates might have been underestimated. However, endotracheal-tube colonisation by Pseudomonas is a well-established phenomenon; a recent study investigating the microbiome of endotracheal tubes showed that the presence of Pseudomonas in the endotracheal tube showed a strong correlation with the poor prognosis of ICU patients. ${ }^{[21]}$

A small percentage of patients also grew MRSA from their tracheal aspirates. Although it has been previously reported, the prevalence of MRSA in hospital-acquired pneumonia is still low. ${ }^{[22]}$

S. maltophilia was grown in two specimens. This opportunistic pathogen primarily infects immunocompromised patients, is often multidrug resistant and is considered to be an independent risk factor for mortality ${ }^{[23]}$ In this study, both patients who had this infection died despite the organism's susceptibility to quinolones and aminoglycosides. The laboratory did not test the susceptibility of this organism for trimethoprim-sulfamethoxazole owing to lack of disks, and hence the susceptibility to this drug could not be ascertained.

Candida spp. and Gram-negative bacilli were the most prominently grown organism from the urine specimens, comparable with previous reports from different regions of the world. ${ }^{[16,24]}$
S. epidermidis was grown from more than $50 \%$ of the blood specimens. This was the most common organism grown from CVC tips as well. It is often regarded as a commensal, unless its growth is associated with infective signs. The high rates may be due to contamination as a result of poor aseptic techniques when obtaining the samples.

Wound swabs from burns and surgical wounds cultured $P$. aeruginosa and MRSA as the most common organisms. In comparison, the most common isolates for wound swabs were Enterobacteriacae, P. aeruginosa and S. aureus in Barbados. ${ }^{[12]}$

In the present study, $P$. aeruginosa showed high sensitivity to quinolones and aminoglycosides. Acinetobacter commonly demonstrates multi-antimicrobial-resistance patterns. In the present study, it showed high sensitivity to quinolones, aminoglycosides and imipenem.

Enterobacteriaceae showed high susceptibility to aminoglycosides and penicillins. However, resistance rates were highest for cephalosporins. Enterobacteriaceae are well known to be resistant to third-generation cephalosporins. ${ }^{[25]}$

The most common antimicrobial used overall in the ICU in this study was cefuroxime, followed by piperacillin/tazobactam and meropenem. Imipenem use was significantly lower than meropenem.

Cefuroxime is often used for surgical prophylaxis, and is continued on the general wards as well. This was shown in a previous study in the same hospital, which revealed a high frequency of prescriptions for cephalosporins on the adult and paediatric medical and surgical wards. ${ }^{[26]}$ In Barbados, the most commonly used antibiotic in their ICU was cefazolin, and this remained consistent during the 3-year period of the study ${ }^{[12]} \mathrm{A}$ study in Brazil also showed similar antimicrobialusage patterns, with high cephalosporin prescription. ${ }^{[27]}$ Such high cephalosporin usage may simply be explained by the fact that there is greater familiarity with the drug, and represents choice based on physician preference rather than being evidence based.

The fact that sensitivity to piperacillin/tazobactam is generally higher than sensitivity to cephalosporins may point towards an inappropriate use of cephalosporins in the study hospital. This is not a problem unique to this hospital; it has also been demonstrated in a tertiary hospital in Port of Spain, the capital city of Trinidad, where there was inappropriate third-generation cephalosporin use in medical, surgical, gynaecological and orthopaedic wards. ${ }^{[28]}$

In our situation, such inappropriate antimicrobial use might also be explained by the absence of an antimicrobial protocol. Also, there is a lack of culture-directed antimicrobial usage, which has implications for the poor de-escalation practices. This results in prolonged, indiscriminate use of broad-spectrum antimicrobials. In addition, this hospital does not have a clinical microbiologist or pharmacist assigned to the ICU to help to guide prescription practices.

The present study had some limitations, the major one being the retrospective design. Missing samples and documentation are commonly encountered in chart reviews, which may impact the findings. In addition, there were some pitfalls unique to the setting, in the guidelines for obtaining samples. For example, tracheal aspirates were the only form of specimens for respiratory infections. Given their low specificity to differentiate colonisation from infection in this sample, bronchoalveolar lavage (BAL) specimens might have been much more informative; however, the ICU did not have a protocol of collecting BAL specimens. This could possibly have led to some overtreatment. Shortage of testing disks for many drugs was also another limitation.

Nevertheless, the present study was able to report the spectrum of microbial growth in the ICU of a tertiary hospital in Trinidad and 
Tobago. It also showed that the overall usage of antimicrobial agents was inappropriate in most instances. This clearly points to a need for improved regular surveillance, the institution of a multidisciplinary team to guide usage and also a need to establish an antimicrobial protocol and guidelines for this ICU.

\section{Conclusion}

Routine microbial surveillance, implementation of an antimicrobial protocol, developing guidelines to regulate the use of antimicrobials and input from infectious-disease specialists are necessary in every ICU setting to contain the development of multidrug-resistant organisms.

Acknowledgements. We acknowledge the contributions made by the staff at the Medical Records Department and the Microbiology Unit at Eric Williams Medical Sciences Complex for their assistance in data collection. Author contributions. All authors contributed equally.

Funding. None.

Conflicts of interest. None.

1. Boev C, Kiss E. Hospital-acquired infections: Current trends and prevention. Crit Care Nurs Clin North Am 2017;29(1):51-65. https://doi.org/10.1016/j.cnc.2016.09.012

2. Chen SY, Wu GH, Chang SC, et al. Bacteremia in previously hospitalized patients: Prolonged effect from previous hospitalization and risk factors for antimicrobial-resistant bacterial infections. Ann Emerg Med 2008;51(5):639-646. https://doi.org/10.106/j.annemergmed.2007.12.022

3. Cardoso T, Almeida M, Carratalà J, et al. Microbiology of healthcare-associated infections and the definition accuracy to predict infection by potentially drug resistant pathogens: A systematic review. BMC Infect Dis 2015;15:565. https://doi.org/10.1186/s12879-015-1304-2

4. Strich JR, Palmore TN. Preventing transmission of multidrug-resistant pathogens in the intensive care unit. Infect Dis Clin North Am 2017;31(3):535-550. https://doi.org/10.1016/j.idc.2017.05.010

5. Bardossy AC, Zervos J, Zervos M. Preventing hospital-acquired infections in low-income and middle-income countries: Impact, gaps, and opportunities. Infect Dis Clin North Am 2016;30(3):805-818. https://doi.org/10.1016/j.idc.2016.04.006

6. Ramsamy Y, Hardcastle TC, Muckart DJJ. Surviving sepsis in the intensive care unit: The challenge of antimicrobial resistance and the trauma patient. World J Surg 2017;41(5):1165-1169. https://doi.org/10.1007/s00268-016-3531-0

7. Li Y, Gong Z, Lu Y, Hu G, Cai R, Chen Z. Impact of nosocomial infections surveillance on nosocomial infection rates: A systematic review. Int J Surg 2017;42:164-169. https://doi. org/10.1016/j.ijsu.2017.04.065

8. Horan TC, Andrus M, Dudeck MA. CDC/NHSN surveillance definition of health care associated infection and criteria for specific types of infections in the acute care setting. Am J Infect Control 2008;36(5):309-332. https://doi.org/10.1016/j.ajic.2008.03.002

9. National Healthcare Safety Network (NHSN). Patient Safety Component Manual. Atlanta: Centers for Disease Control and Prevention, 2017. https://www.cdc.gov/nhsn (accessed 4 August 2017).

10. Rosenthal VD, Maki DG, Graves N. The International Nosocomial Infection Control Consortium: Goals and objectives, description of surveillance methods and operational activities. Am J Infect Control 2008;36(9):1-12. https://doi.org/10.1016/j.ajic.2008.06.003
11. Rosenthal VD. Device associated nosocomial infection in limited resource countries: Findings of the International Nosocomial Infection Control Consortium. Am J Infect Control 2008;36(10):712. https://doi.org/10.1016/j.ajic.2008.10.009

12. Hariharan S, Nanduri SB, Moseley HSL, et al. Spectrum of microbes and antimicrobial resistance in a surgical intensive care unit, Barbados. Am J Infect Control 2003;31(5):280-287.

13. Tamakoshi K, Toyoshima H, Yatsuya H, et al. White blood cell count and risk of all-cause and cardiovascular mortality in nationwide sample of Japanese - results from the NIPPON DATA90. Circ J 2007;71(4):479-485.

14. Chen YY, Chou YC, Chou P. Impact of nosocomial infection on cost of illness and length of stay in intensive care units. Infect Control Hosp Epidemiol 2005;26(3):281-287. https://doi. org $/ 10.1086 / 502540$

15. Jeganathan N, Yau S, Ahuja N, et al. The characteristics and impact of source of infection on sepsisrelated ICU outcomes. J Crit Care 2017;41:170-176. https://doi.org/10.1016/j.jcrc.2017.05.019

16. Ding JG, Sun QF, Li KC, et al. Retrospective analysis of nosocomial infections in the intensive care unit of a tertiary hospital in China during 2003 and 2007. BMC Infect Dis 2009;9:115. https://doi. org/10.1186/1471-2443-9-115

17. Sader HS, Castanheira M, Flamm RK. Antimicrobial activity of ceftazidime-avibactam against Gram-negative bacteria isolated from patients hospitalized with pneumonia in US medical centers, 2011 to 2015. Antimicrob Agents Chemother 2017;61(4):e02083-16. https://doi org/10.1128/AAC.02083-16

18. Djordjevic ZM, Folic MM, Jankovic SM. Distribution and antibiotic susceptibility of pathogens isolated from adults with hospital-acquired and ventilator-associated pneumonia in intensive care unit. J Infect Public Health 2017;S1876-0341(17)30028-X (e-pub. ahead of print). https://doi. org/10.1016/j.jiph.2016.11.016

19. Azzab MM, El-Sokkary RH, Tawfeek MM, Gebriel MG. Multidrug-resistant bacteria among patients with ventilator associated pneumonia in an emergency intensive care unit, Egypt. East patients with ventilator associated pneu

20. Bhadade R, Harde M, deSouza R, More A, Bharmal R. Emerging trends of nosocomial pneumoni in intensive care unit of a tertiary care public teaching hospital in Western India. Ann Afr Med 2017;16(3):107-113. https://doi.org/10.4103/aam.aam_7_17

21. Hotterbeekx A, Xavier BB, Bielen K, et al. The endotracheal tube microbiome associated with Pseudomonas aeruginosa or Staphylococcus epidermidis. Sci Rep 2016;6:36507. https://doi. org/10.1038/srep36507

22. Lollar DI, Rodil M, Herbert B, Burlew CC, Pieracci FM. Empiric methicillin resistan Staphylococcus aureus coverage in the early ventilator associated pneumonia window: If and when. Surg Infect (Larchmt) 2016;17(2):187-190. https://doi.org/10.1089/sur.2014.159

23. Nseir S, Di Pompeo C, Brisson H, et al. Intensive care unit-acquired Stenotrophomona maltophilia: Incidence, risk factors, and outcome Crit Care 2006;10:R143. http://doi.org/ 10.1186/ cc5063

24. Aubron C, Suzuki S, Glassford NJ, Garcia-Alvarez M, Howden BP, Bellomo R. The epidemiology of bacteriuria and candiduria in critically ill patients. Epidemiol Infect 2015;143(3):653-662. https://doi.org/10.1017/S0950268814000934

25. Oliveira MC, Oliveira CR, Gonçalves KV, Santos MS, Tardelli AC, Nobre VA Jr. Enterobacteriaceae resistant to third generation cephalosporins upon hospital admission: Risk factors and clinical outcomes. Braz J Infect Dis 2015;19(3):239-245. https://doi.org/10.1016/j. bjid.2015.01.006

26. Hariharan S, Pillai G, McIntosh D, et al. Prescribing patterns and utilization of antimicrobial drugs in a tertiary care teaching hospital of a Caribbean developing country. Fundam Clin Pharmacol 2009;23(5):609-615. https://doi.org/10.1111/j.1472-8206.2009.00713.x

27. Dos Santos EF, Lauria-Pires L. Patterns of antibacterials use in intensive care units. Rev Bras Ter Intensiva 2010;22(2):144-152. https://doi.org/10.1590/S0103-507X2010000200008

28. Pinto Pereira LM, Phillips M, Ramlal H, et al. Third generation cephalosporin use in a tertiary hospital in Port of Spain, Trinidad: Need for an antibiotic policy. BMC Infect Dis 2004;4:59. https://doi.org/10.1186/1471-2334-4-59

Accepted 28 August 2017. 